\title{
Histological, Histochemical and Immunohistochemical Alterations Induced in Rat's fetalskeletal Muscle Fibres and Skin Intoxicated Maternally with Carisoprodol (Somadril Compound)
}

\author{
Hanaa A. Abd El-Gawwad* and Asmaa M. Abd El-Azez \\ Zoology Department, Faculty of Science, Al-Azhar University, Egypt \\ *Corresponding author: Hanaa A. Abd El-Gawwad, Email: hanaa_khedr99@yahoo.com
}

\begin{abstract}
Background: skeletal muscle relaxants contain a diverse mix of agents with different buildings and tools for achievement. These agents are classified as antispasmodics or antispasmodic agents, one of them is the drug carisoprodol. Objective: the current study aimed to investigate the possible effect of somadril compound on the skeletal muscle fibres and skin of fetuses maternally treated with carisoprodol.Material and methods: 30 pregnant female rats were categorized into 3 groups equal in number ten for each group, the first one (Control group ) ten pregnant female rats were administrated oral doses of distilled water; rats of the second group (S1) were administrated oral doses of carisoprodol in distilled water equivalent to $10.8 \mathrm{mg} / 100 \mathrm{~g}$ body weight/ day and rats of the third group (S2) were administrated oral dose of carisoprodol in the distilled water equivalent to $21.6 \mathrm{mg}$ $/ 100 \mathrm{~g}$ body weight/day. Rats of S1 and S2 were administrated oral dose of carisoprodol for 15 days from the $6^{\text {th }}$ day to the $20^{\text {th }}$ day of gestation. Many histological, histochemical and immunohistochemical studied were done to detect the alterations in the fetal skeletal muscles and skin. Results: fetal skin and skeletal muscle tissues of both treated groups showed many changes compared to the control group. Conclusion: treatment of the pregnant rats with carisoprodol led to numerous pathological changes in the fetal skin and skeletal muscle fibres.
\end{abstract}

Keywords: carisoprodol, fetuses, immunohistochemical, skin, histology, histochemical,skeletal musclefibres.

\section{INTRODUCTION}

Somadril is the brand name for carisoprodol and it is a muscle relaxant. It is used to relax certain muscles in the body, relieving stiffness, discomfort, pain and to treat spasms. It acts on the central nervous system which in turn signals to the muscles the desired effect. It is administrated in tablets. Somadril can cause several side effects in the heart, skin, breast etc., because of the common side effects of drowsiness dizziness, somadril does indeed cause their effects where you are taking it, if it avoid driving operating machinery or any other exercises that require mental or physical alertness;in addition somadril depressed the central nervous system ${ }^{(1)}$. In Egypt, carisoprodol is available as somadril compound which contains carisoprodol 200mg, paracetamol $160 \mathrm{mg}$ and caffeine $32 \mathrm{mg}$. It is a pre-described drug produced by Mina Pharm for Pharmaceutical Regulations by the decision of the Minister of Health and Population No 172 of the year 2011, while the meprobamate the active metabolite of Carisoprodol $^{(2)}$ is a schedule iii controlled substance on anti-drug law no. 182 of the year 1960. The drug is found in markets by diversity names (Soma, Sodol, Somalgit, Vanadam, Sonoma, Scutamil, Carisoma, Somacid, Mio Relax, Relacton-C, Relaxibys, Rela and Soridol) ${ }^{(3)}$, Skeletal muscle relaxants contain a diverse mixture of proxies with different buildings and instrument of achievement. These proxies are categorized as antispasticity or antispasmodic proxies; one of them is carisoprodol drug ${ }^{(4)}$.It essentially acts as a skeletal muscle relaxant and it is thought to slab inter-neuron action by stimulating GABA-A receptors in descendent reticular creation and spinal rope ${ }^{(5)}$. In a previous study,carisoprodol treatment of the pregnant rats caused severe variations in the esophageal tissue of fetuses such as : highly thickened mucosal layers with lots of vacuolated cells and highly distorted submucosal layer, discontinuous and ruptured muscle fibres in the muscularis after administrated of carisoprodol ${ }^{(7)}$. Also, lung of treated rats with somadril showed congested alveolar septa, highly congested blood vessels which contained haemolysed blood cells,highly thickened alveolar septa, reduced alveolar sacs with thickened and corrugated epithelial layer of the bronchioles ${ }^{(\mathbf{8})}$.

Hepatic tissue of pregnant rats administrated somadril showed many alterations such as highly congested and dilated central veins and blood sinusoids, highly distorted portal areas, hepatocytes were vacuolated and contained pyknotic nuclei ${ }^{(9)}$. Also, administration of rats with somadril showed many alterations in testis such as vacuolation, degeneration in spermatozoa within the lumen and los of normal architecture of the seminiferous tubules ${ }^{(\mathbf{1 0})}$.

\section{MATERIAL and METHODS \\ Drug:}

Carisoprodol drug was obtained as tablets of a union product containing carisopordol $200 \mathrm{mg}$, paracetamol $160 \mathrm{mg}$ and caffeine $32 \mathrm{mg}$. It is obtained from Mina Pharma for Pharmaceuticals and Chemical Industries, Cairo. Egypt. 


\section{Animals Model:}

This current study was carried out on adult Albino rats (Sprague Dawleystrain ) with an average weight of 140-180 g. They were kept under normal conditions of temperature, humidity and normal light cycle and supplied with food and water. The Estrus phase was performed according to Taylor ${ }^{(11)}$. Ratio of females to males was 2:1 and they were put in a single cage overnight for fertilization. The presence of vaginal plug means that breeding had occurred and the hat was reflected zero-day of pregnancy ${ }^{(\mathbf{1 2})}$.

\section{Experimental design:}

Thirty rats were categorized into three groups as follows:

Group 1: ten pregnant female rats were administrated distilled water orally and known asC.

Group 2:- ten pregnant rats were taken oral doses of carisopordol equal to $10.8 \mathrm{mg} / 100 \mathrm{gm}$ body weight (low dose).

Group 3:- ten pregnant rats were taken oral doses of carisopordol equal to $21.6 \mathrm{mg} / 100 \mathrm{gm}$ body eight (high dose).

This administration was done every day for 15 days from the $6^{\text {th }}$ to $20^{\text {th }}$ day of pregnancy ${ }^{(\mathbf{1 3})}$.

\section{Histological and histochemical techniques:}

Fresh small pieces of fetal skin and skeletal muscles were fixed $10 \%$ neutral buffer formalin solution for the histological and histochemical studies, then washed with distilled water, dehydrated in alcohol, cleared with xylene, embedded in paraffin wax, cuttingwas done with the microtome at $4 \mathrm{um}$ in thickness and stained with Harris hematoxylin and eosin ${ }^{(14)}$.Masson's trichrome stain forwas used for detecting collagen fibres, thepolysaccharides were detected by PAS (Periodic acid chiffreagent )$^{(\mathbf{1 5})}$.Protein was detected by the mercury promo phenol method ${ }^{(16)}$ and Congo red stain for amyloid $\beta$ $\operatorname{protein}^{(17)}$.

\section{Immunohistochemistry examination:}

Immunohistochemical detection of Caspase-3 was performed by using an anti-caspase-3 primary antibody (Labvision, Neomarkers, USA) according to the protocol described by AbdelWahabandMetwally ${ }^{(18)}$ using a streptavidin-biotin system.Positive reaction for Caspase- 3 was visualized as the brown coloration of the cytoplasm in the treated tissues.

\section{Ethical approval:}

The study was approved by the Ethics Board of AlAzhar University and an informed written consent was taken from each participant in the study. Statistical analysis

Statistical analyses were performed using analysis of variance (ANOVA) according to the method Jaeger, $2008^{(19)}$. The data were proceeded and analyzed using SPSS software (statistical analysis for social sciences, version 23 ). Significant differences between treatment means were determined by the student $t$-test. Data were presented as means $\pm \mathrm{SE}$ and $\mathrm{P} \leq 0.05$ was considered statistically significant.

\section{RESULTS \\ The Histopathologic Investigation: 1- Skin:}

Foetal skin of the control group showed normal epidermis and dermis with underling subcutaneous tissues (Fig. 1 A).The epidermal layer was normal with Malpighian, stratified squamous epithelial cells and keratin layers (Fig.1B). The dermal layer was composed of connective tissue, blood vessels and hair follicles, the latter were normally growing with organized epidermal, cortical and medullary cell layers (Fig. 1C\&D). The skin of foetal rats of group S1 showed epidermal apoptotic changes, cytoplasmic granulation and actively dividing mesenchymal cells( Fig.2 B). Some of the hair follicles were affected with disorganized epidermal, cortical and medullary cell layers (Fig 2C\&D).Subepidermal focal muscular morphogenesis was observed (Fig. 2A). On other hand, the fetal skin of group S2 showed many changes included, deformed keratin layer, disarrangement of the epidermal cells, apoptotic changes, cytoplasmic granulation and dilatation of dermal blood vessels (3A,B \&C).Some of hair follicles were atrophied with disorganized epidermal, cortical and medullary cell layers ( Fig.3 D).Masson's trichrome stained sections of foetal rat skin of the control group showed normal distribution of collagen fibres ( Fig. 4 A ), while in S1and S2 groups showed increased collagen fibers in the epiderma land dermal tissues especially hair follicles with hemorrhages and dilated capillaries (Fig.4 B\&C).

\section{2- Muscles:}

Normal structure of muscles with apparent longitudinal striation was detected in fig. (5A). Disorganization and partial disappearance of striations of muscle fibres with edematous changes in interstitial connective tissues were observed in both $\mathrm{S} 1$ and S2 groups ( Figs 5 B \&C). In addition, numerous dystrophic changes were observed in the fetal muscle fibresof group S2 such as vacuolated, degenerate with numerous pyknotic nuclei (Fig.5\&C). Masson's trichrome stained sections of fetal rat skeletal muscle of the control group showed normal distribution of collagen fibers (Figure 6A). Increased collagen fibres were observed in between and around the skeletal muscle fibres of fetuses related to S1 and S2 groups (6 $\mathrm{B} \& \mathrm{C})$. Increased collagen fibres were also observed in and around the walls of the dilated blood vessels (Fig.6C). 


\section{The Histochemical Investigation:}

\section{Skin:}

Fetal skin of the control group stained with PAS showed a slight deposition of polysaccharides in the dermal and hypodermal muscle fibres with less staining affinityin the epidermis and hair follicles. Poor staining affinity was observed in the keratin layer ( Fig. 7A, histogram 1).On the other hand, examination of fetal skin of group S1 and S2 indicated more deposition of polysaccharides in the epidermal apoptotic cells, deformed hair follicles cells and in between and inside the hypodermal connective tissue cells (Fig.7B\&C, histogram 1).

Skin of the control fetuses showed dense staining affinity of proteinic materials in the epidermal layers especially keratin, hair follicles with less staining affinity in the dermal layer (Fig. $8 \mathrm{~A}$, histogram 1). On the other hand, examination of fetal skin of group S1 and S2 showed decreased total protein content especially in the epidermal keratin layerand dermal layer( Fig. 8 B\&C, histogram 1).

The skin of control fetuses showed a slight deposition of amyloid beta-protein (Fig. 9 A, histogram 1).Foetal skin of group S1 and S2 showed increased amyloid beta-protein (Fig. $9 \&$ B, histogram 1).

\section{Muscles :}

Normally stained polysaccharides were noticed in the fetal skeletal muscle fibres of the control group ( Fig..10A, histogram 2). On other hand, highly increased polysaccharides were observed in the fetal skeletal muscle fibres of S1 and S2 groups especially in the interstitial connective tissue ( Fig.10B\&C, histogram 2 ).Figure 11 showed normal distribution of total proteins in the fetal skeletal muscle fibres of the control group. On the other hand, examination of fetal muscle fibres of group S1 and S2 showed decreased density of total protein content ( Fig. 11 B\&C, histogram 2).Fetal muscle fibres of the control group showed a slight deposition of amyloid beta-protein ( Fig.12A, histogram 2). On the other hand, examination of fetal muscle fibres of groups S1 and S2 showed increased deposition in amyloid beta-protein( Fig.12 $\mathrm{B} \& \mathrm{C}$, histogram2 ).

\section{Immunohistochemical Observation :}

Skin and muscular tissues of rat's fetuses of the control group showed weekly expression to Caspase -3 immunostaining (Fig. 13\&14).On the other hand,fetal skin and muscle fibres of groups S1and S2 showed intense expression for Caspase -3 immunostaining determined by the dense brown stain compared tothe control group (Fig. 13\&14 B\&C).
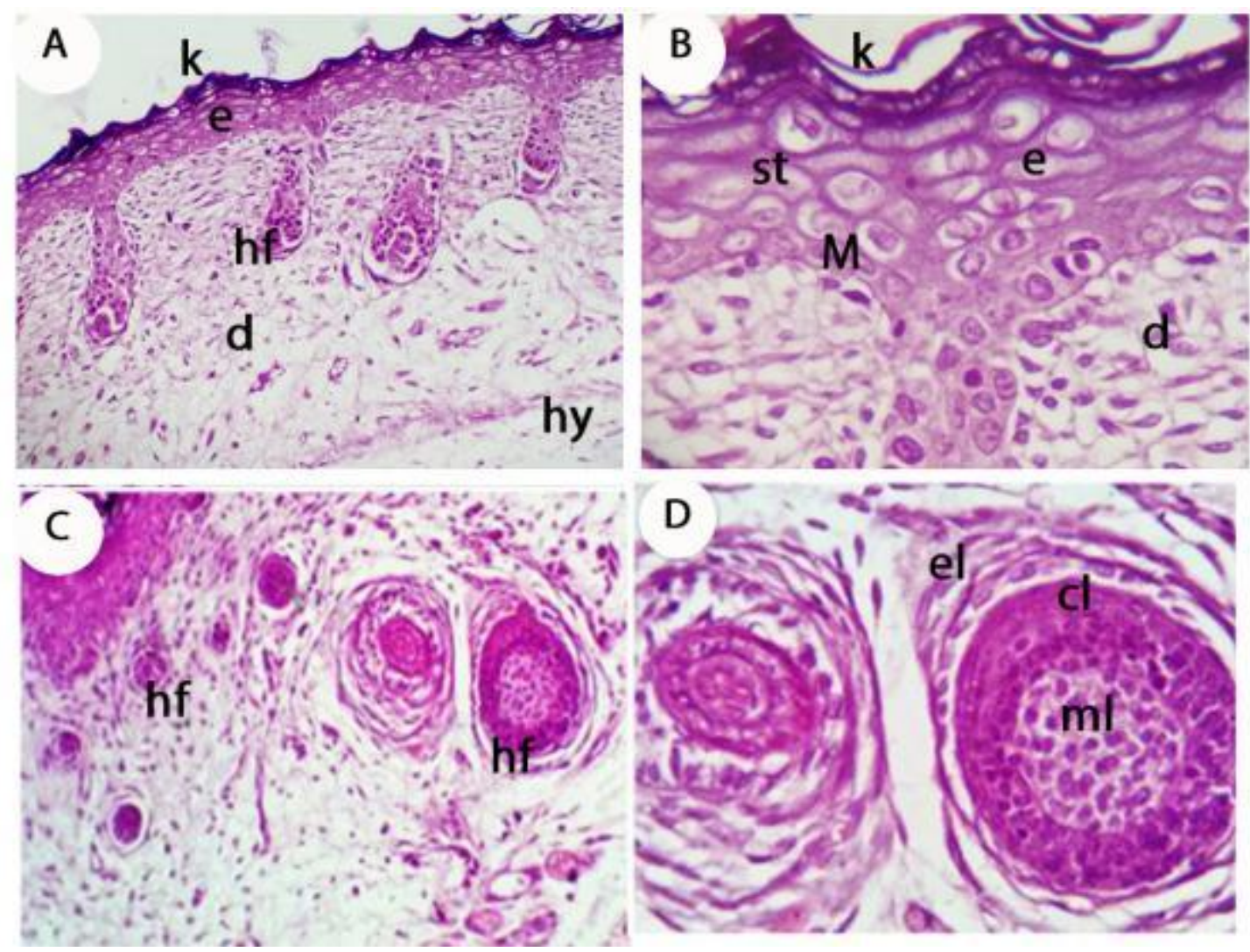

Fig.1 A-D- Photomicrographs of sections in fetal skin of the control group stained with hematoxylin and eosin, A\&B show normal structure of epidermis (e), dermis (d) and hypodermis ( hy). Notice :epidermal structure with epidermal gradual keratinization ( $\mathrm{k}$ ), stratified squamous epithelial cells (st) and Malpighian cell layer (M); C\& D show dermal layer with normal hair follicles (hf) with organized epidermal (el), cortical (cl) and medullary (ml) cell layers. (AX100,B\&D X 400,CX200). 

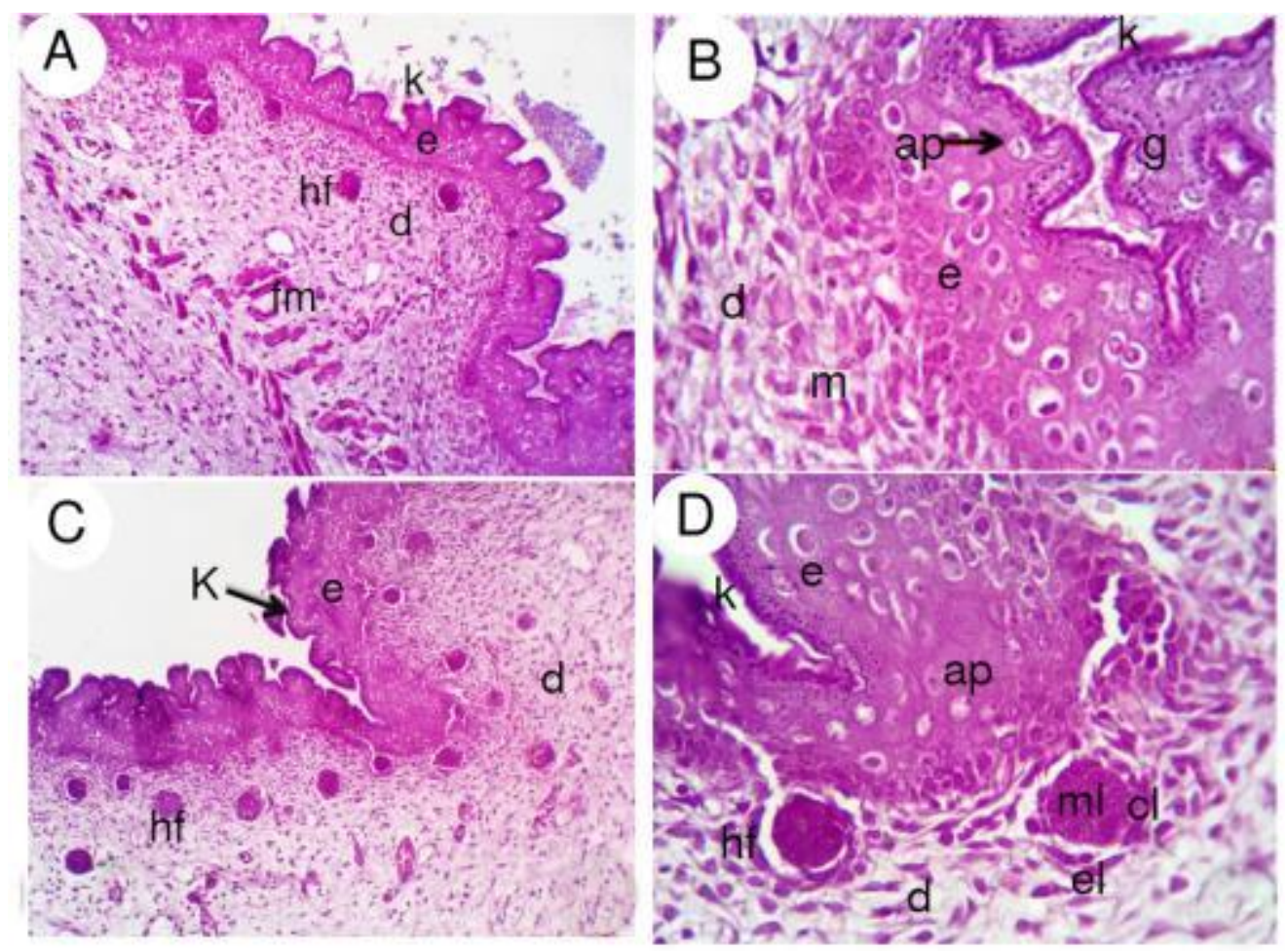

Fig.2 A-D- Photomicrographs of sections in fetal skin of S1group stained with hematoxylin and eosin, show irregular keratin (k) and epidermal layer (e) with apoptotic changes( ap) and cytoplasmic granulation( $\mathrm{g}$ ) ,dermal layer (d) with actively increased mesenchymal cells $(\mathrm{m})$, atrophied hair follicles (hf) with disorganized epidermal (el), cortical (cl) and medullary (ml) cell layers, hypodermal focal muscular morphogenesis (fm).( A\&CX200, B\&D X 400)

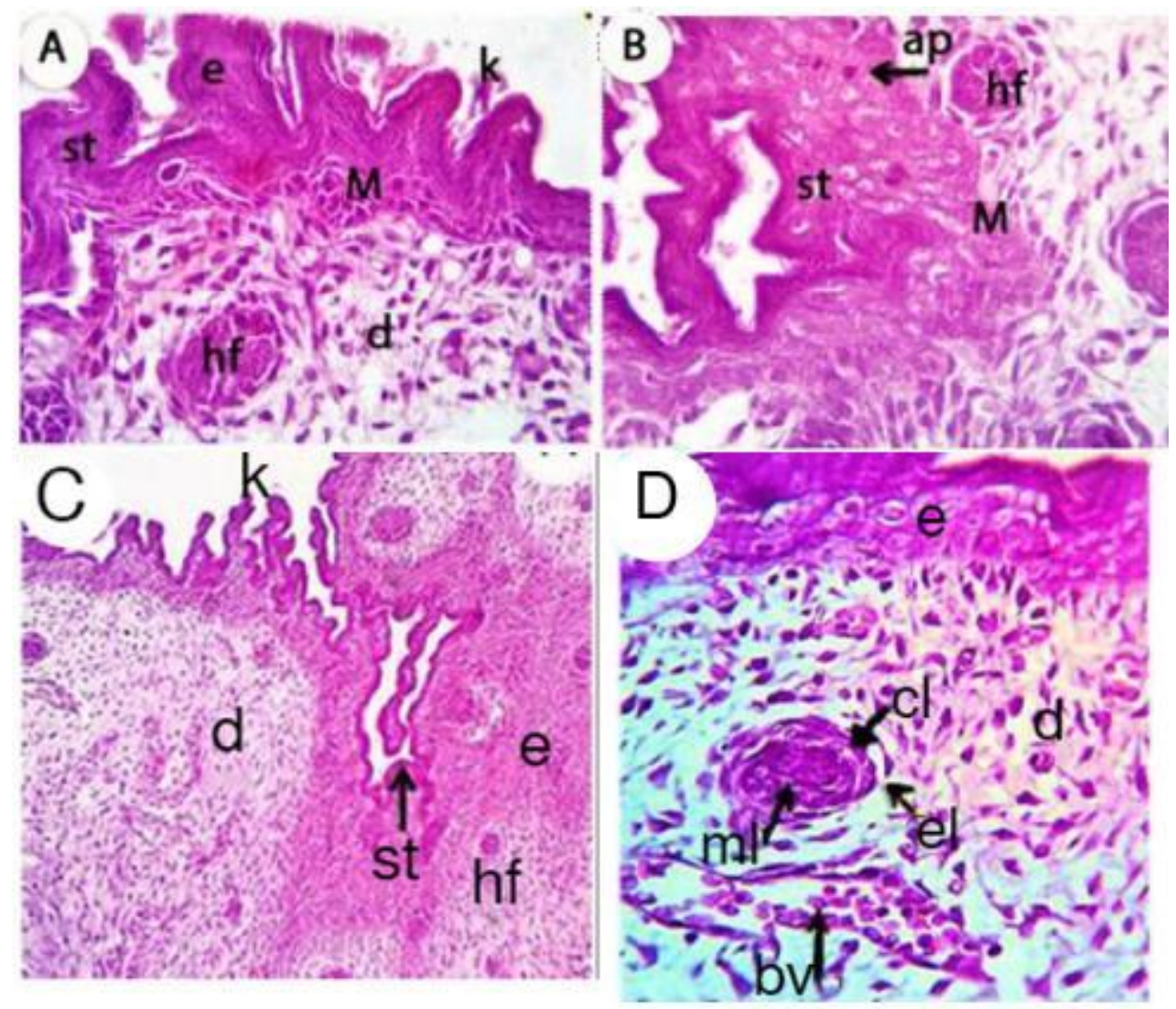

Fig.3 A-D- Photomicrographs of sections in fetal skin of S2 group stained with hematoxylin and eosin, show highly thickened and irregular keratin (k) and epidermal layers (e), disturbed and irregular Malpighian layer (M), apoptotic changes (ap), granulation (g) and vacuolation (v) of the stratified squamous epithelial cells (st), atrophied hair follicles (hf) and dilated blood vessels (bv). (A\&DX 200 ,BX 400 -CX 100). 

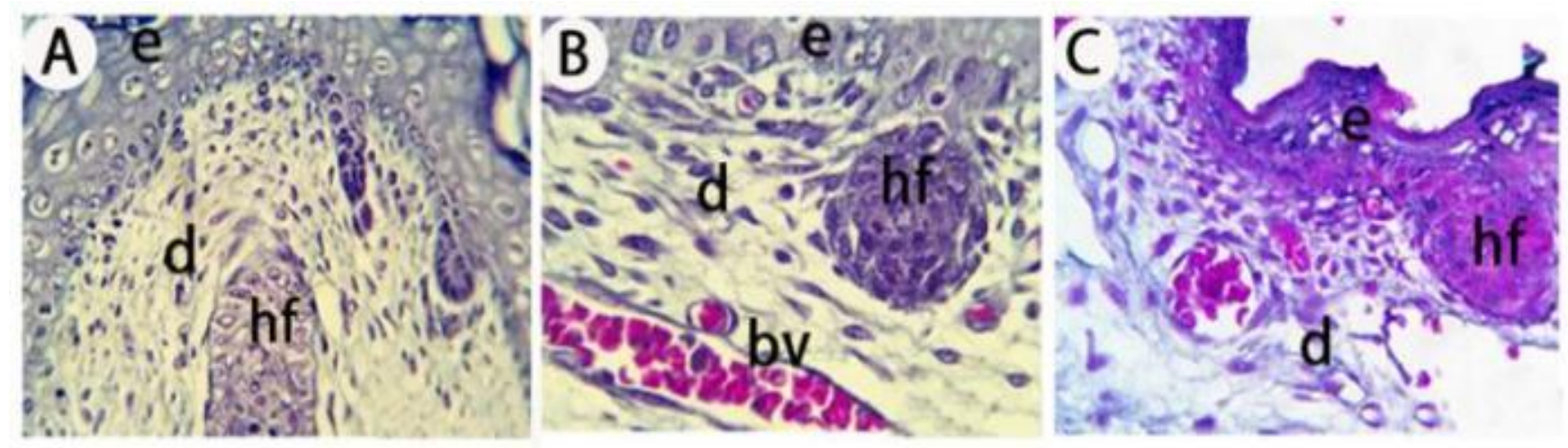

Fig.4 A-C- Photomicrographs of sections in fetal skino $\mathrm{f}$ the control and treated groups stained with Masson's trichrome, A- control group shows normal distribution of collagen fibresin the keratin layer(k), epidermis(e), basement membrane, dermis (d) and hair follicle (hf) ,B- S1group shows increased collagen fibres in the dermal layer (d) and dilated blood vessels , C- S2 group shows highly increased collagen fibres in the epidermal ( e) and dermal layers (d) especially in the keratin (k) and stratified squamous epithelial ( st) layers and in the cortex and medulla with marked degenerated hair follicles (hf) .( A,B\&C X 200)

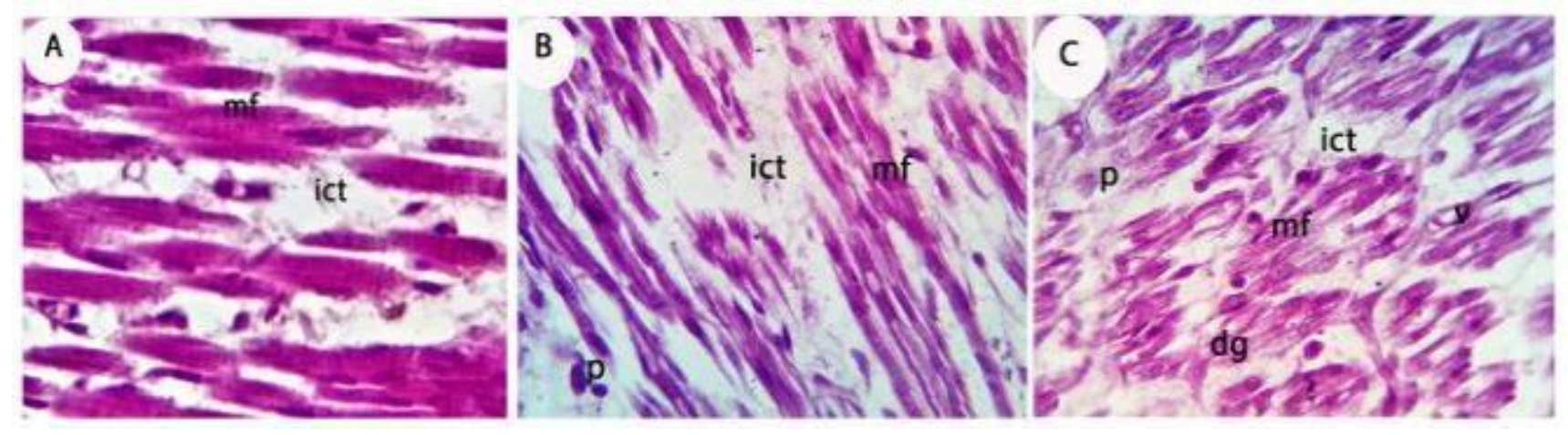

Fig.5 A-C- Photomicrographs of sections in fetal muscles of control and treated groups stained with hematoxylin and eosin, A- The control group shows normal muscle fibres (mf) with apparent longitudinal striation and interstitial connective tissue (ict), B- S1 group shows partially discontinuous and disturbed muscle fibres (mf) with pyknotic nuclei (p), C- S2 group showsd isorganization and disappearance of striations with interstitial edematous changes ( ict) and highly vacuolated (v), pyknotic (p) and degenerated muscle fibres (dg). $(\mathrm{A}, \mathrm{B} \& \mathrm{C} X \mathrm{400})$
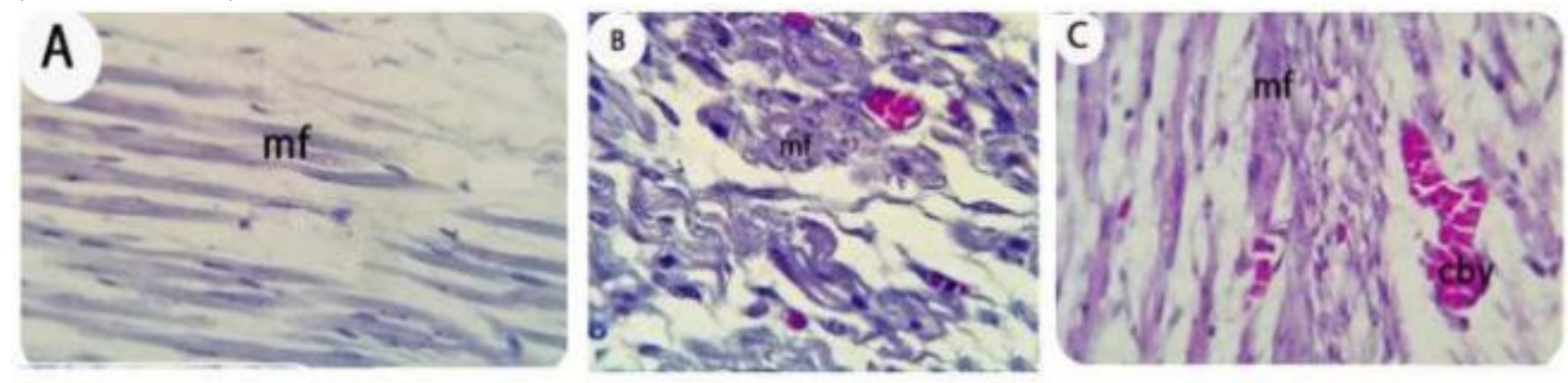

Fig.6 A-C- Photomicrographs of sections in fetal musclesof the control and treated groups stained with Masson's trichrome, A- control group shows normal distribution of collagen in the muscle fibres (mf), (B) S1 group shows increased collagen fibers in between the muscle fibres (mf), (C) S2 groups hows highly increased collagen fibres in the muscle fibres in and around wall of the congested blood vessels. (A,B\&C X 200 ). 


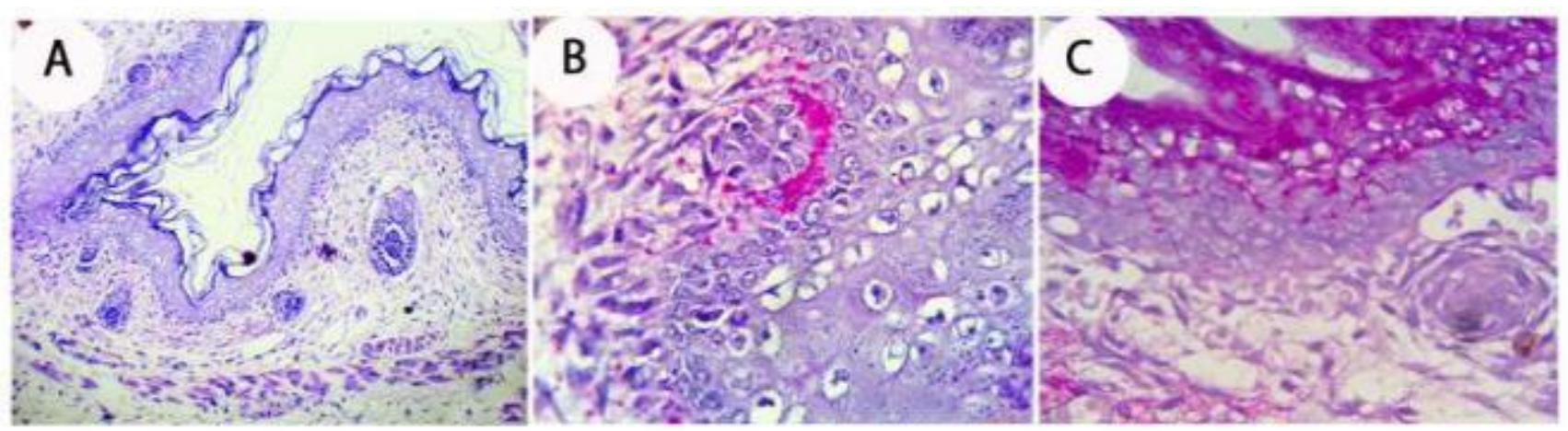

Fig.7 A-C- Photomicrographs of sections in fetal skin of the control and treated groups stained with PeriodicAcid Schiff technique (PAS) with hematoxylin as a counter stain for detecting polysaccharides, Acontrol group shows normal distribution of polysaccharides, B\&C- Low dose S1 and high dose S2 groups of somadril show increased polysaccharides ( A,B\&C X 200 )
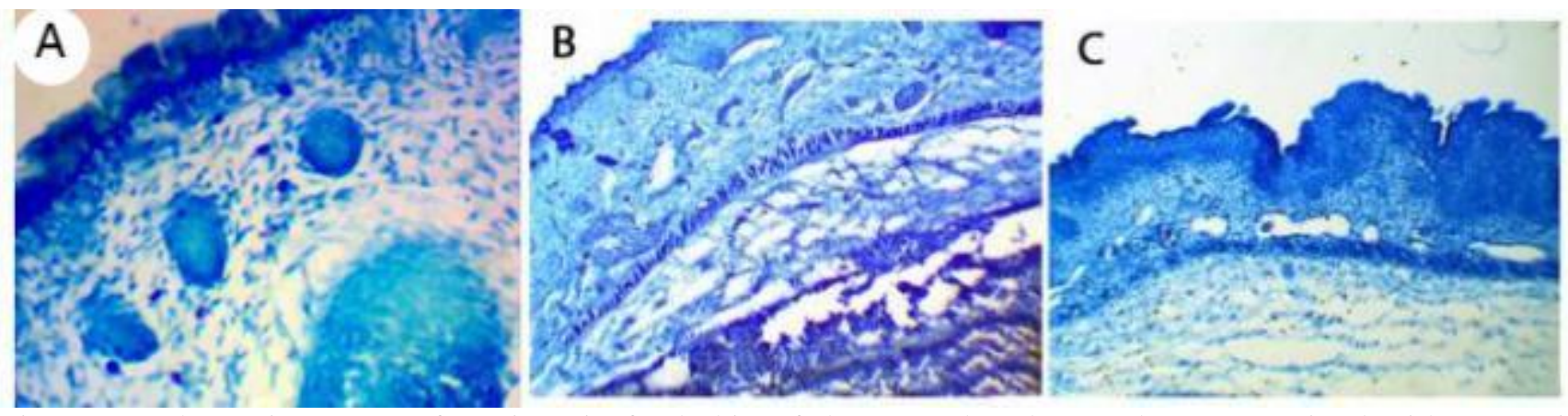

Fig.8 A-C-Photomicrograps of sections in fetal skin of the control and treated groups stained with mercuric bromophenol blue for detecting total proteins. A- control group shows normal distribution oftotal proteins . Dense staining affinityty is observed in the epidermal layer, keratin and hair follicles(X200). B\&C- Low dose $\mathrm{S} 1$ and high dose S2 groups of somadril showing moderately decreased stain ability of total protein in the keratin and epidermal layers.(X200).

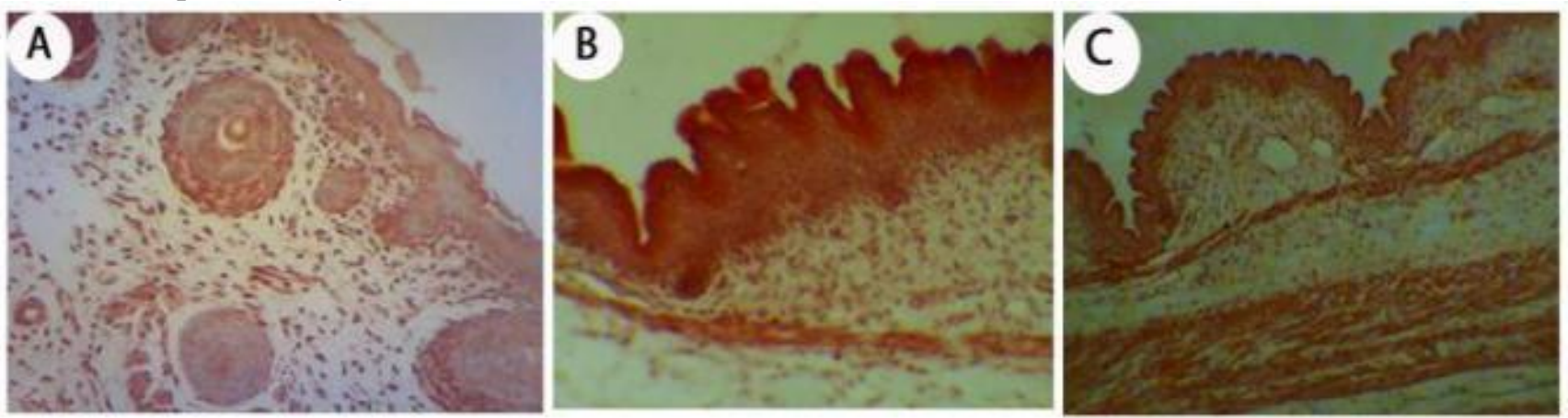

Fig.9 A-C-Photomicrographs of sections in fetal skin of the control and treated groups stained by Congo red for detecting amyloid B protein. A-The control group shows faintly stained amyloid -Bprotein.(X200).B\&C-Low dose S1 and high dose S2 groups of somadril show densely stained amyloid B protein especially in the epidermal layer.(X200).
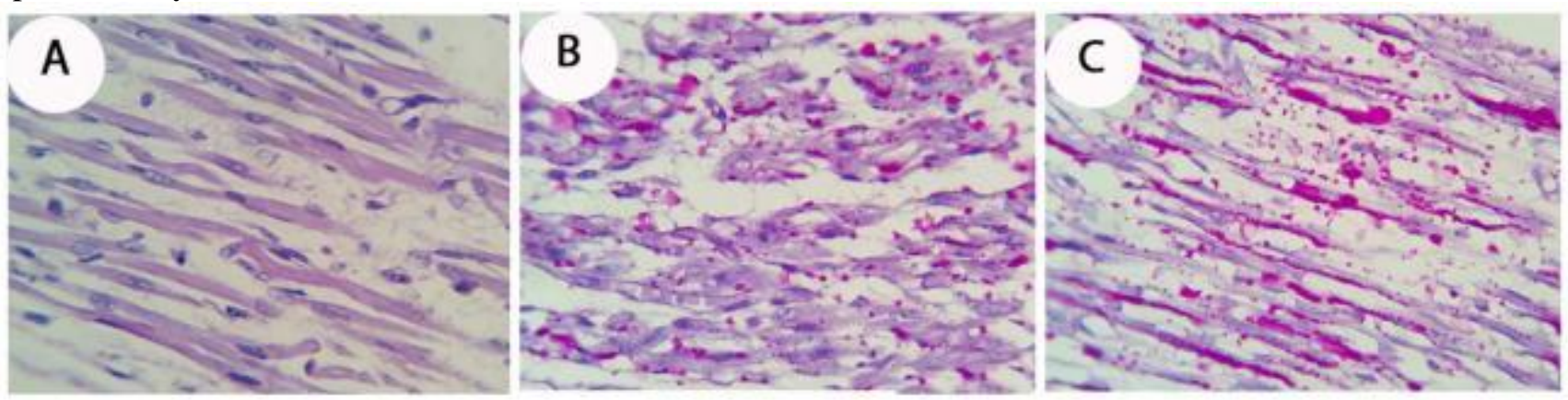

Fig.10 A-C- Photomicrographs of sections in fetal skeletal muscles of the control and treated groups stained with periodic acid Schiff technique (PAS) with haematoxylin as a counter stain for detecting polysaccharides, AControl group shows normal distribution of polysaccharides. B\&C-Low dose S1 and high dose S2 groups of somadril showing increased staining affinity ofr polysaccharides in the skeletal muscles (A,B\&C X 200 ). 


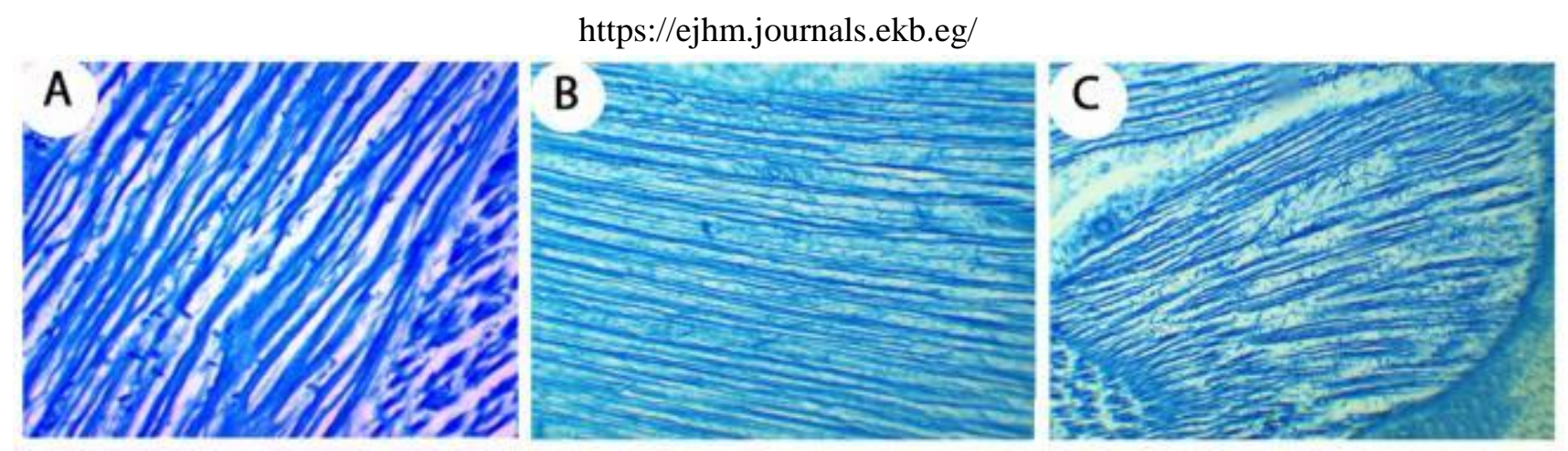

Fig.11 A-C- Photomicrographs of sections in fetal skeletal muscle fibres of the control and treated groups stained with mercuric bromophenol blue for detecting total proteins. A-Control group shows normal proteinic content in the muscle fibres.(X200). B\&C-Low dose S1 and high dose S2 groups of somadril showing decreased distribution of total protein in the muscle fibres.(X200)
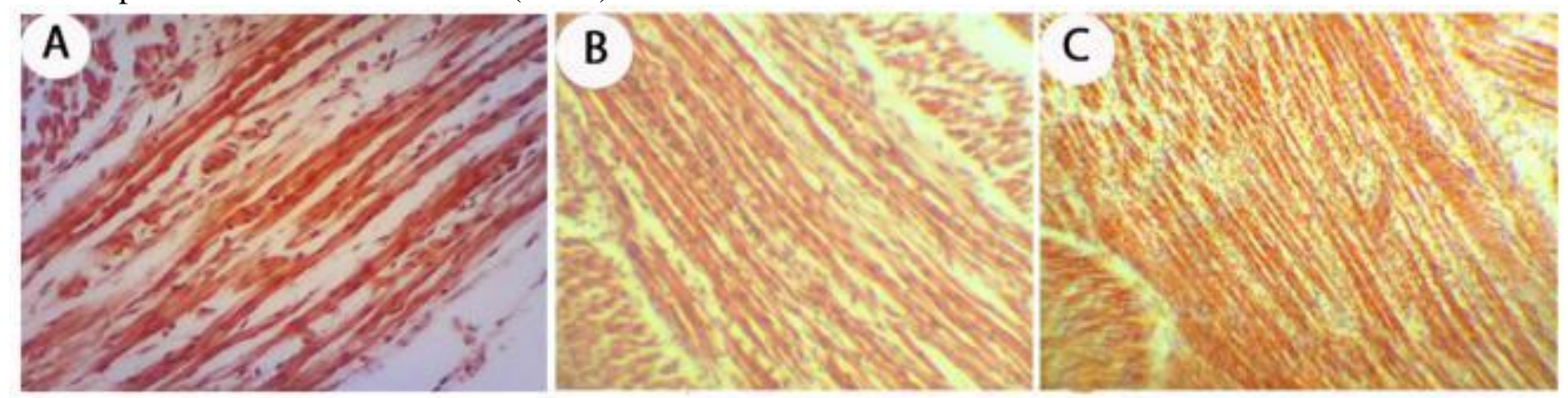

Fig. 12 A-C- Photomicrographs of sections in fetal muscle fibers of the control and treated groups stained by Congo red for detecting amyloid B protein. A- The control group shows faintly stained amyloid -B protein.(X200). B\&C-Low dose S1 and high dose S2 groups of somadril show densely stained amyloid B protein in the muscle fibres.( X200)
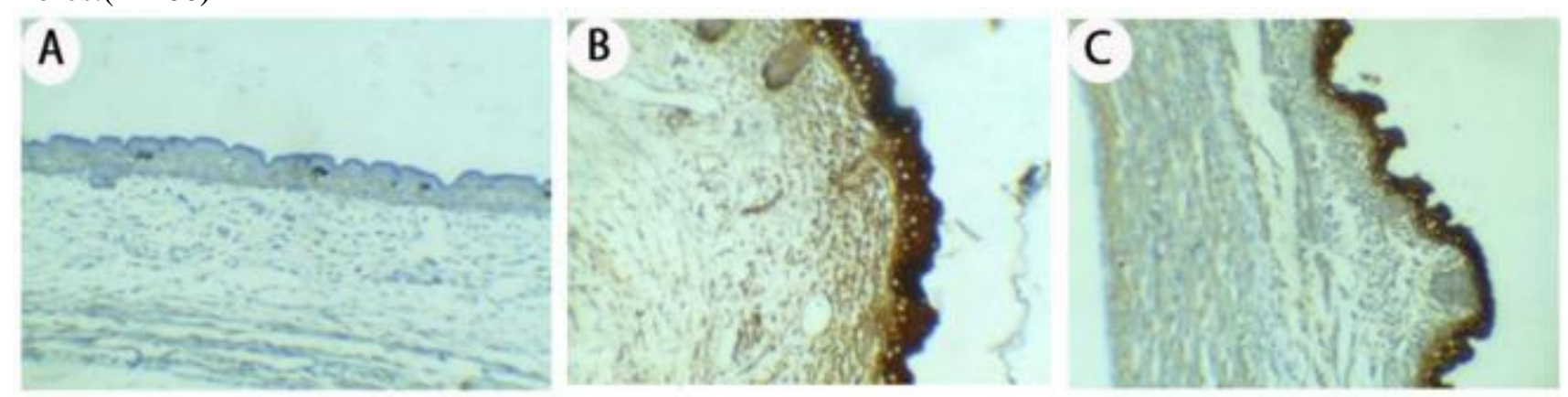

Fig. 13 A-C- Photomicrographs of sections in fetal skinof the control and treated groups stained with caspase-3immune-staining, A- The control group showing positive weak expression for caspase -3(X200), B\&C- Low dose $\mathrm{S} 1$ and high dose $\mathrm{S} 2$ groups of somadril showing intense expression of caspase-3 in the epidermis, keratin and hair follicles which is indicated by dense brown staining affinity and moderate expression of caspase -3 in the dermal and subcutaneous tissues.(X200).

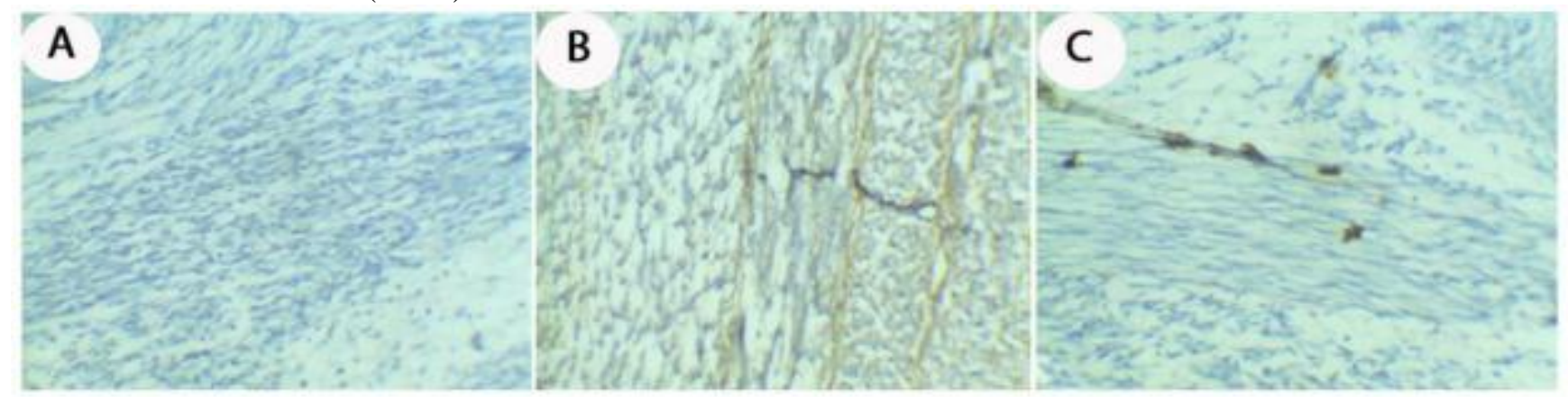

Fig. 14 A-C- Photomicrographs of sections in fetal muscle fibers of the control and treated groups stained with caspase-3- immune-staining. A- The control group showing weak expression for Caspase -3(X200). B\& C- Low dose S1 and high dose S2 groups of somadril showing moderate to intense expressionof caspase -3 in the degenerated muscle fibres.(X200) 


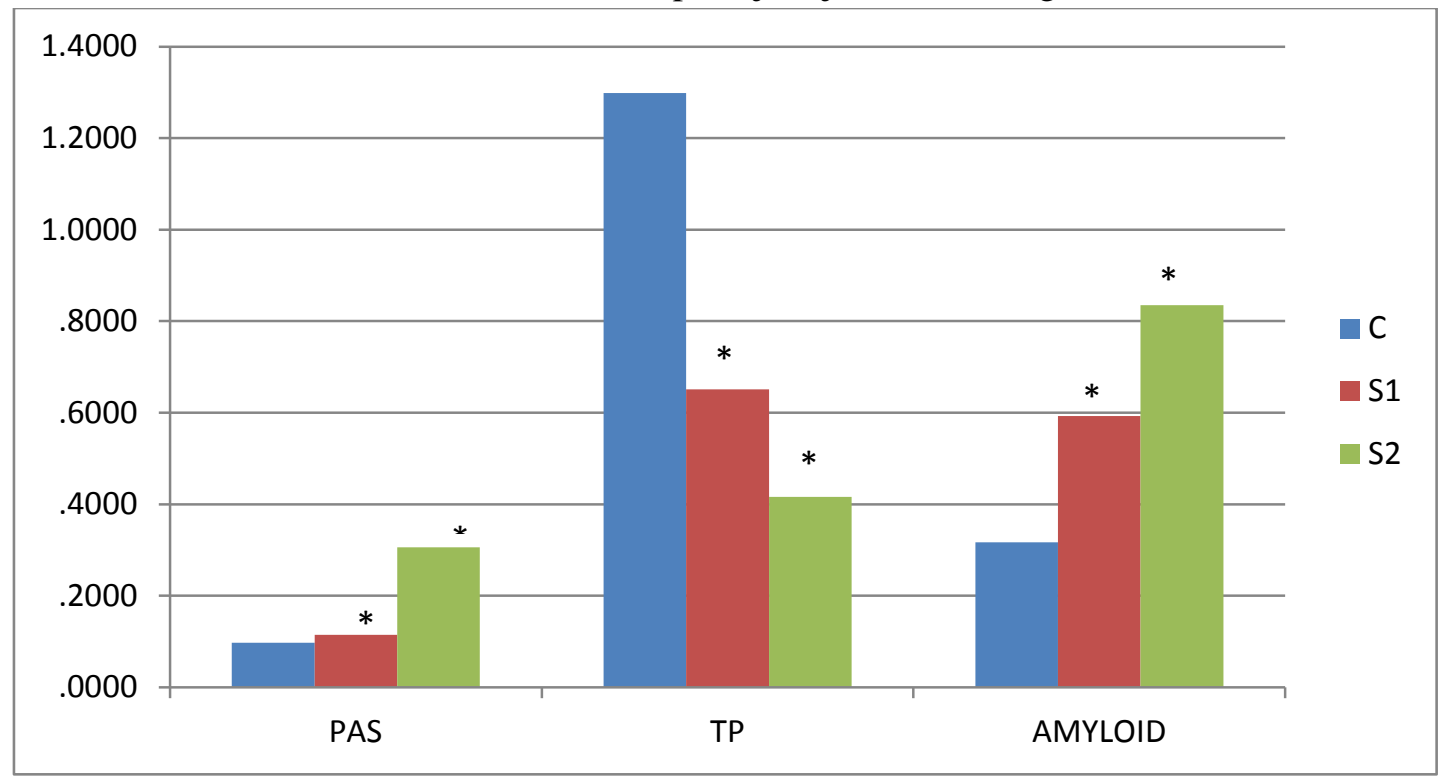

Histogram 1-The optical density of PAS+ve materials, total protein and amyloid beta protein in the skin of the control and treated experimental groups

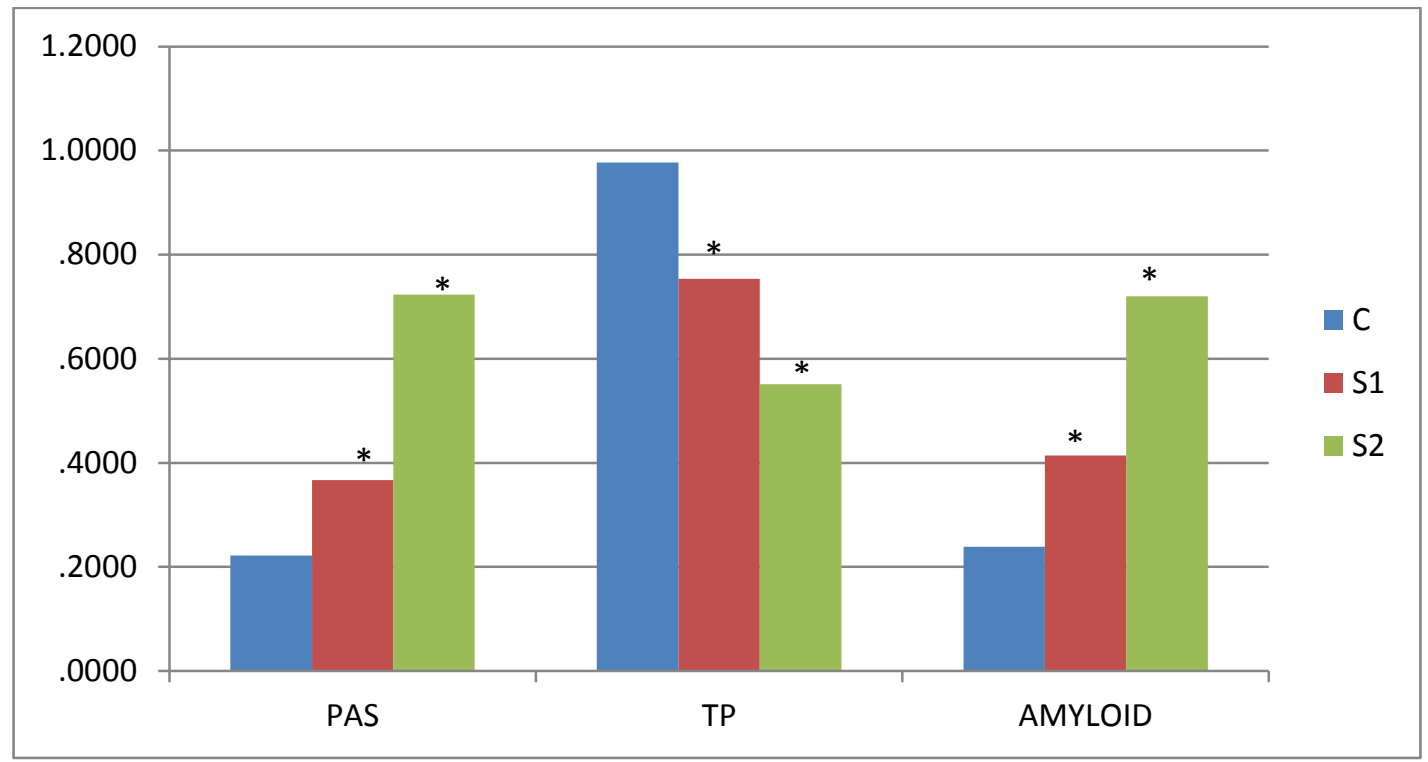

Histogram 2-The optical density PAS +ve materials, total protein and amyloid beta protein in the fetal muscle fibres of the control and treated experimental groups.

\section{DISCUSSION}

\section{Skin:}

Problems of tension in skeletal muscles and backbone pain can be resolved by carisoprodol ${ }^{(\mathbf{3 4})}$. Carisoprodol produces muscle relaxation by blocking intraneuronal activity in the descending reticular fibres and spinal cord ${ }^{(29)}$. Rapid gastro absorption results in peak carisoprodol plasma concentrations of $4-7 \mathrm{mg} / \mathrm{ml}$ within $2-4 \mathrm{~h}$ and the beginning of action is within $30 \mathrm{~min}$ of ingestion ${ }^{(9,30)}$. In the liver, carisprodol is metabolized to meprobamate ( the primary active metabolites) which is excreted via the renal and non-renal pathways. In the pregnant rat, carisoprodol can cross the placenta ${ }^{(20)}$ and congenital abnormalities were occurred ${ }^{(9)}$.The same author added that carisoprodol was excreted in breast milk at concentrations two to four times that in maternal plasma.skin is probably the path for excretion of risky substances occurred in the environment.skin is one of the pathways for removing risky substances occurred in the environment. Also, the skin is considered as a mirror for the internal body and its healthy look gives a good idea about the state of the organs ${ }^{(30)}$.In the present work, fetuses of mothers treated with carisoprodol showed many alterations in the skin of groups S1 and S2 compared to the control group. the skin of fetal rats of group S1 showed epidermal apoptotic changes, cytoplasmic granulation and activity increased mesenchyme cells. Some hair follicles were affected with disorganized epidermal, cortical and medullary cell layers while, S2 treated group had deformed keratin layer, disarrangement of epidermal cells, dilation of dermal blood vessels, apoptotic changes and cytoplasmic granulation. These investigations agree with those of Anwar et al. ${ }^{(10)}$ and Abouel-Magd $^{(9)}$ since treatment with somadril 
showed testicular vacuolation and degeneration, reduced spermatozoa in the lumen and loss in normal architecture of the seminiferous tubules.

In this work highly increased collagen fibres was detected in the epidermal and dermal layers especially hair follicles, hemorrhage and dilated capillaries were also observed in the treated fetal groups S1 and S2 compared to the control fetal groups. This investigation agrees with those of Abdelhafez and AbdRabou ${ }^{(20)}$ who treated rats with somadril and they noticed highly increased collagen fibres in the renal tissues of pregnant rats of group S1 and S2 especially in the brush borders and basement membranes of the convoluted tubules.

Hassan et $\boldsymbol{a l} .{ }^{(\mathbf{2 1})}$ stated that increase collagen fibers may lead to protection from a toxic substance. increased collagen deposition maylead to oxidative stress that stimulates the expression of the genes involved in collagen biosynthesis ${ }^{(22)}$. In the current study a slight deposition of polysaccharides was realized in the dermal and hypodermal muscle fibres of the control group while, the fetal treated group had a high deposition of polysaccharides in the epidermal apoptotic cells. These results agree with those of AbdRabou $^{(7)}$ who found increased PAS (+ve) materials in the fetal esophagus tissuesin S1 and S2 groups. Results of the current study showed an increase in staining affinity of total protein in the epidermal layer, keratin layer and hair follicle of treated fetal groups S1 and S2. increased staining affinity of total protein may be attributed to the generation of reactive oxygen species stress and consequent oxidative stress ${ }^{(23)}$ this agree with results of Abd-ElHady and Aljalaud ${ }^{(24)}$ who declared that the increase in staining affinity of the total protein of lung tissue following toxicity may be attributed to increased red blood cells in the congested blood vessels. The current work recorded a significant increase in the amyloid B- protein deposits in the fetal skin of groups S1 and S2 especially in the keratin layer of the epidermis, these results agree with those of Abouel-Magd $^{(9)}$ who found thatthe increased deposition of amyloid B protein in hepatocytes, blood cells, necrotic areas and also in the hemolysed blood cells of fetal liver tissue of groups S1 and S2. Kadawakietal. ${ }^{(25)}$ showed that amyloid deposition was associated with dysfunction and resulting generation of reactive oxygen species which can initiate a signaling pathway leading to apoptosis.

\section{Muscles:}

In the present study, carisoprodol treatment of pregnant rats with two doses $\mathrm{S} 1$ and $\mathrm{S} 2$ induced dystrophic changes on fetal skeletal muscle fibres which increased in their severity in a dose-dependent manner. Numerous histological changes were represented by disorganization with the disappearance of striations of muscle fibres, edematous changes between fibres, dilated blood vessels, some fibroblast were vacuolated, degenerated and contained pyknotic nuclei. These investigations agree with those of Abouel-Maged ${ }^{(9)}$, Anwar et al. ${ }^{(10)}$ and Ali et al. ${ }^{(28)}$ who found that treated adult male rats with somadril at $200 \mathrm{mg} / \mathrm{kg}$ led to destruction of the histological architecture of the seminiferous tubules, testicular vacuolation and degeneration and reduced spermatozoa within the lumen of the testis. Treatment of carisoprodol at doses 10.8 and $21.6 \mathrm{mg} / \mathrm{kg}$ caused degenerative changes in the lung, esophagus and liver tissues of the pregnant rats and their fetuses ${ }^{(7-9)}$.

Also, the results of the present study are supported by results of Abouel-Maged ${ }^{(9)}$ who declared that carisoprodol is taken up by the central nervous system within forty seconds after intravenous injection of mice and distributed throughout the body with ten minutes. The highest concentration of carisoprodol was found in the lung, liver pituitary gland, heart, adrenal gland followed by the blood and skeletal muscles. The same author added that radioactivity was uniformly distributed throughout the fetuses of pregnant mice after15 minutes of carisoprodol administration. These results agree with the present results and have proven that somadril has destructive effects on fetal tissues. In the present study, increased collagen fibres were detected in between and around the skeletal muscle fibres and around wall of the dilated blood vessels of fetuses related to $\mathrm{S} 1$ and $\mathrm{S} 2$ groups in comparison with the control group.

AbdRabou $^{(7)}$ noticed increased collagen fibres in esophageal tissues of fetuses of rats maternally treated with carisoprodol during pregnancy. Normally, collagen is essential for formation of muscles, skin, ligaments and blood vessels. The increased collagen may lead to an increase in the defense reaction against toxic materials ${ }^{(31)}$. Increased collagen deposition may be due to encourage of expression of genes involved in collagen biosynthesis due to oxidative stress ${ }^{(9,20)}$. Results of the current study revealed an increase of polysaccharides in the fetal skeletal muscle fibres of S1 and S2 groups in a dose-dependent manner as compared to the control group. Such increasein polysaccharides may be due to the increase of red blood corpuscles after toxicity ${ }^{(8)}$.

In the present study, examination of fetal muscle fibres of groups S1 and S2 showed decreased density of total protein content. AbouEl Naga andAbdRabou ${ }^{(32)}$ revealed that the decrease in protein content may be due to decreased ribosomes or rapture of cellular organelles. In addition, a decrease of protein content may be due to degenerated tissues and lysosomal membranes disruption by the effect of toxicants which leads to releasing of their hydrolytic enzymes in the cytoplasm which causing lysis of the target materials with it ${ }^{(33)}$.

In the present study, amyloid-beta protein increased in the fetal skeletal muscles in S1 and S2 sets compared to the control set.These results agree with those of Abdelhafez and AbdRabou ${ }^{(20)}$ who found increased amyloid-beta protein in the liver tissue 
of the fetal rat maternally treated with carisoprodol. The same authors added that amyloids are insoluble fibrous protein and they result from polypeptides and nearly 18 unsuitably folded forms of protein that present normally in the body.These misfolded constructions alter their good configuration and they erroneously interact with each other or other cell components forming insoluble amyloid fibers.Also, the present results agree with the study of AbdRabou and Alotibi ${ }^{(34)}$ who found an increase in amyloid-beta protein in the fetal ileum tissues of the pregnant rat treated with carisoprodol. The same authors stated that the abnormal accretion of amyloidbeta protein in the tissues may play an important function in numerous neurodegenerative syndrome and cause amyloidosis. In nerve cells, these amyloid proteins can accumulate forming amyloid plexus may cause blocking a signal path which results in Alzheimer's disease. This aggregation is related to dysfunction in mitochondria and causes the generation of ROS which can promote a signaling path leading to apoptosis.In this study, fetal skin and skeletal muscles of the control group showed weak expression to caspase- 3 while, the treated groups S1 and S2 showed intense deposition for caspase 3 immunostaining which indicated severe apoptosis. These investigationsare constant with other studies on CNS deposits as Glooetal. ${ }^{(26)}$ who stated that acetaminophen drug has been demonstrated to cause cell apoptosis and necrosis.

\section{CONCLUSION}

It can be concluded that using somadril drug during the pregnancy period caused many alterations and hazardous changes in the skin and skeletal muscle of fetuses because the mother treated with somadril during the pregnancy period.

\section{REFERENCES}

1. Lawrence F, Iona J, Nancy B(2008): Neonatal Dermatology. $2^{\text {nd }} \quad$ Ed,.Saendens Elsevier, Pp. 576.https://www.elsevier.com/books/neonataldermatology/eichenfield/978-1-4160-3432-2

2. Bramness J, Buajordet I, Skurtveit S (2008): The role of phamaco-epidermisological studies in the market withdrawal of Crisopordol (Somadril) in Europe.Norsk.Epidemiology,18 (2):167-172.

3. Reeves R., Burke R , Kose S (2012):Crisoprodol update on abue potential and Legal status.Southern Medical Journal,105(11):616-623.

4. Witenko C, Moorman C, Motycka K et al. (2014): Considerations for the appropriate use of skeletal muscle relaxants for the management of acute low back pain.Partitioning and Transmutation, (6): 427-435.

5. Ralph L, Look W, Wheeler S et al. (2008): Doubleblind, placebo-controlled trial of carisoprodol $250-\mathrm{mg}$ tablets in the treatment of acute lower-back spasm. Current Medical Research and Opinion, 24 (2):551- 558.

6. Rust G, Hatch R , Gums J (1993):Carisoprodol as a drug of abuse. Arch. Fam. Med., 2:429-435.
7. Abd-Rabou M (2019): Histological and histochemical alteratos induced in rats fetal eosophageal tissues intoxicated maternally with carisporodol. Egyptian Journal of Hospital Medicine, 75(2):2254-2260.

8. Emam N (2019): Maternal and fetal toxicity of carisopordol drug (Somaril compound) on pulmonary tissues of albino Rats. J. Biology and Life Science, 1: 7297.

9. Abouel-Magd M (2018): Maternal and fetal toxicity of carisoprodol. The Egyptian Journal of Hospital Medicine, 71(1): 2322-2350.

10. Anwar K, Salih L, Abdulkareem S (2017):Histophysiological study of some parts of organs treated with somadril drug. Journal of Raparin University, 4 (11):3344.

11. Taylor P (1986): Handling of the reproductive cycle and mating. In: Practical Teratology. Taylor, P. (Ed.)., Academic Press, London, Pp: 3-21.

12. Nazari Z, Nabiuni S, Ghaffari $M$ et al. (2017): Gestational diabetes induces pancreatic $\beta$-cells apoptosis in adult rat offspring. Int. J.Morphol., 35: 16-20.

13.Paget G , Barnes J (1964): Interspecies Dosage Conversion Scheme In Evaluation Of Results and Quantitative Application In DifferentSpecies.Laurence D $\mathrm{R}$ and Bacharach A L (Eds.).Academic Press, London and USA. Pp: 160-162.

14. Bancroft Jand Gamble $M$ (2002): Theory and Practice of Histology Techniques. $5^{\text {th }}$ ed. Churchil Livingstone, London. Pp: 150-152.

15. Hotchkiss R(1948):Amicro-chemical reaction resulting in he staining of polysaccharide structure in fixed tissue preparation. Arch.Biochem.,16:131-132

16. Mazia $D$, Brewer $P$, Alfert $M$ (1953): The cytochemical staining and measurement of protein with mercuric bromophenol blue. Biol. Bull., 104(1): 57-67.

17. Valle $S$ (1986): Special stains in microwave oven. J.Histotechnol., 9: 237-248.

18. Abdelwahab B, Metwally M (2015): Clozaprine induced cardiotoxicity: role of oxidative stress, tumour,necrosis, factor alpa and NF.ק. Cardiovascular Toxicology, 15:355-365.

19.Jaeger T (2008): Categorical data analysis: Away from ANOVAs (transformation or not) and towards logit mixed models. J.Mem.Langu., 59(4): 434-446.

20.Abdelhafez H, Abd Rabou M (2020):Carisoprodol (somadril) indued histological and histochemical changs in renal cortex of pregnant rats and their fetuses the Egypt. J. Histology,34(3):835-848

21.Hassan H,Ghaly E, EL-Nashar A et al. (1988):Histochemicl study on some organs of rats fed rape seed and cotton see oils . Egypt. J.Histolo., 11(2):247-252

22.Guter G,Turkozor Z,Ozgur E et al. (2009): protein oxidatonunderextremly low frequency electric field in Guinea pigs -Effect of N- acetyl -L- cysteine treatment. Gen. Physiol. Biophys., 28: 47-55.

23.Sumen S, Rodriguez O, Winters $T$ et al. (2013): Therapeutic and space radiation exposure of mouse brain causes ipaired DNA repair response and premature senescence of chronic oxidant production.AGING., 5(8): 607-622.

24.Abd-Elhady A, Aljaloud N (2015): Therapeutic effects of olive leaf extract or bone marrow mesenchymal stem cells against lung. Damage induced $\mathrm{n}$ male albino rats 
exposed to gamma radiation. The Egyptian Journal of Hospital Medicine, 61: 685-699.

25. Kadawaki H (2005): Amyloid beta induces neuronal ASKI activation. Cell Death Diff., 12(1): 19-24.

26. Gloor Y, Schvartz D, Samer C (2019): Old problem, new solutions: biomarker discovery for acetaminophen liver toxicity. Export.Opin. Drug Metab.Toxicol., 15(8): 659-669.

27. Reeves R, Burke $\mathbf{R}$, Kose $\mathbf{S}$ (2012):Carsoprodol: Update on Abuse Potential and Legal status. The Southern Medical Association, 105 ( 11) :619-623.

28. Ali K, Salih L, Abdulkareem S (2017):HistoPhysiological Study of some Parts of Organs Treated with Somadril Drug. Journal of Raparin University,4: (11): 33-44.

29. Inoue Y(2002): Medical economics - Hepatitis Cvirus infectionand cost - effectiveness ofInterferon. Thera. Res., 24: 57-67.
30.Alkaabi O (2008):Electomagnatic field effect on skeletal muscles and skin of albino rat embryos histological and histochemical studies. The Egyptian Journal of Hospital Medicine, 33: 479- 491.

31. Mansour M, Ibrahim M , Mahmoud H (2013): The possible protective role of bone marrow transplantation against alternations induced by gamma radiation on heart of pregnant albino rats and their fetuses. .J Biology and Life Science,4(1):247-272.

32.Abou El Naga N, Abd Rabou M (2012): The possible protective role of bone marrow transplantation on irradiated mothers and their fetuses. Stem Cell, 3(3): 830 .

33. Abdel-Meguid N, Chmaisse H, Abouzeinab N(2012): Silymarin ameliorates Cisplatin : induced hepato toxicity in rats : histopathological and ultrastructure studies. J. Biol. Sci., 13: 463-479.

34. AbdRabou M, Alotaibi M (2021):Toxicity of Somadril compound on fetal ileum tissues of albino rats.Pakistan J. of Biol. Sci., 24(1): 72-79. 